\title{
Influencia de la temperatura sobre la asimetría de pilancones en ambiente granítico. Aplicación de un modelo de regresión lineal
}

\author{
Manuel García-Rodríguez, Abel Sánchez-Jiménez, Antonio Murciano, Blanca Pérez-Uz,
} Mercedes Martín-Cereceda

\begin{abstract}
Manuel García-Rodríguez
manu.garo@ccia.uned.es

Departamento de Ciencias Analíticas, Facultad de Ciencias Universidad Nacional de Educación a Distancia (UNED). Paseo Senda del Rey 9. 28040, Madrid (España).
\end{abstract}

\section{Abel Sánchez-Jiménez \\ Antonio Murciano \\ Departamento de Matemática Aplicada (Bio- matemática), Facultad de Ciencias Biológicas. Universidad Complutense de Madrid (UCM). 28040, Madrid (España).}

\section{Blanca Pérez-Uz}

Mercedes Martín-Cereceda

Departamento de Microbiología III, Facultad de Ciencias Biológicas. Universidad Complutense de Madrid (UCM). 28040, Madrid (España).
BOL. SOC. GEOL. MEX. 2017

VOL. 69 NO. 2

P. $479-494$

\section{RESUMEN}

Las pilas representan un tipo de forma presente en casi todos los ambientes climáticos. El trabajo estudia el papel de los ciclos térmicos como un agente importante del modelado y asimetría que presentan las paredes de las pilas en un clima Mediterráneo templado - frío. El estudio se ha realizado en el macizo granítico de la Pedriza de Manzanares, zona protegida de gran valor ambiental, incluida en el Parque Nacional de la Sierra de Guadarrama (Madrid, España). $\mathrm{El}$ análisis de la variabilidad térmica mediante modelos de regresión periódica múltiple, pone de manifiesto la influencia de los ciclos diario y anual en función de las orientaciones norte y sur de las paredes de las pilas. Un modelo matemático de regresión lineal muestra cómo la variabilidad térmica diaria influye en la alteración de las paredes de las pilas, generando superficies planas o de concavidad más o menos pronunciada. El trabajo también establece relaciones entre el grado de alteración de las diferentes partes de las pilas, con la presencia de líquenes y dureza relativa de la roca. Los resultados avalan la hipótesis de relación causal entre la variabilidad térmica y alteración de las paredes de las pilas según su orientación norte o sur.

Palabras clave: pilas, modelo matemático, meteorización del granito, La Pedriza de Manzanares.

\section{ABSTRACT}

Weathering pits represent a kind of geological form present in almost all climatic environments. This manuscript explores the role of thermal cycles as important agents in the modelling and asymmetry of the walls of weathering pits in a temperate-cold Mediterranean climate. This study has been developed in the granite massif from La Pedriza in Manzanares which is a protected area, with a great environmental value, allocated in the National Park Sierra de Guadarrama (Madrid, Spain). The analysis of thermal variability was approached using periodic multiple regression that showed the influence of daily and annual cycles depending on the north and south orientation of the pit walls. A mathematical model of linear regression revealed the influence of the daily thermal variability on the alteration of the pit walls generating flat surfaces or a more or less pronounced concavity. This study also established relationships between the alteration degree of different areas of the pits with the lichen presence and with the relative hardness of the rock. The results support the hypothesis of a causal relationship between thermal variability and the shaping of the weathering pits depending on their north or south orientation.

Keyreords: gnamma, mathematical modelling, weathering granite, La Pedriza de Manzanares. 


\section{Introducción}

\subsection{ORIGEN Y MEGANISMOS DE FORMAGIÓN DE LAS PILAS}

Las pilas o pilancones, se utilizan ambos términos dependiendo de su tamaño relativo, son cavidades de morfología cóncava que se desarrollan sobre la superficie de las rocas por un proceso de meteorización y erosión. Las pilas pueden tener formas variadas, siendo las más frecuentes de aspecto circular, o ligeramente elongadas. La anchura y profundidad de las pilas puede variar desde pocos centímetros a varios metros (Twidale y Corbin, 1963). Aunque se trata de una morfología muy típica de rocas graníticas (Vidal Romaní, 1985), son también frecuentes en areniscas (Alexandrowicz, 1989), calizas (Gutiérrez Elorza e Ibáñez, 1979), basaltos (Dorn, 1995), gneises y otras rocas. Casi todos los factores que participan en la formación de pilancones tienen relación con el clima. Su origen ha sido sugerido por diversos autores en prácticamente todos los ambientes climáticos: en regiones tropicales (Branner, 1913), en ambientes áridos (Maclaren, 1912; Wilhelmy, 1964; Twidale y Bourne, 1975), periglaciares (Matthes, 1930), templados (Smith, 1941) y mediterráneos (Domínguez-Villar, 2007).

Twidale y Bourne, (1975) consideran que las pilas se inician bajo el suelo por procesos químicos y continúan desarrollándose al salir a la superficie. El inicio de estas cavidades en condiciones de enterramiento puede atribuirse, por ejemplo, a la alteración de enclaves más solubles que la roca caja, o bien por irregularidades en el frente de alteración. $\mathrm{Su}$ origen también puede explicarse por la presencia de fracturas (Figura 1a) que representan planos de debilidad por los que penetra el agua (Twidale y Bourne, 1976; Uña, 1999). Vidal Romaní, (1983) sugiere que en algunos casos su formación puede estar relacionada con la concentración de cargas en ambientes relativamente profundos. Aunque son muchas las teorías sobre el origen de las pilas, quizá la idea más aceptada sobre su inicio hace referencia a la meteorización por efecto del agua al quedar retenida en depresiones de la roca (Figura 1b). La permanencia de agua en las zonas depri- midas por más tiempo que en las zonas periféricas, hace que se meteoricen más rápidamente (Domínguez-Villar, 2007; Domínguez-Villar et al., 2009). (Figura 1).

No hay un criterio unitario al respecto en cuanto a la velocidad de formación de estas morfologías (Hall y Phillips, 2006; Domínguez-Villar y Jennings, 2008). Domínguez-Villar, (2007) describe pilancones de evolución subaérea en el Sistema Central español que evolucionaron desde el final del Pleistoceno superior, dónde la disolución mineral es muy dinámica y su efecto es visible tras varios meses de observación de un mismo pilancón. El agua está considerada como el elemento principal para que se pongan en marcha algunas reacciones químicas, como la hidrólisis, la hidratación y oxidación (Twidale y Bourne, 1975), que son las que más afectan a los minerales presentes en los granitos. La efectividad de la meteorización química estará directamente influenciada con la humedad ambiental, grado de saturación de la roca (Mol y Viles, 2012) y tiempo de permanencia del agua en contacto con la roca (Brady y Walther, 1990; Blum y Stillings, 1995).

Estudios de Difracción de Rayos X de los minerales el granito sobre pilancones en el Sistema Central español, llevados a cabo por Domínguez-Villar (2007), señalan que el índice de disolución más elevado corresponde a las plagioclasas primero, seguido de los feldespatos potásicos, que forman la mayoría de los cristales que sobresalen en las pare-

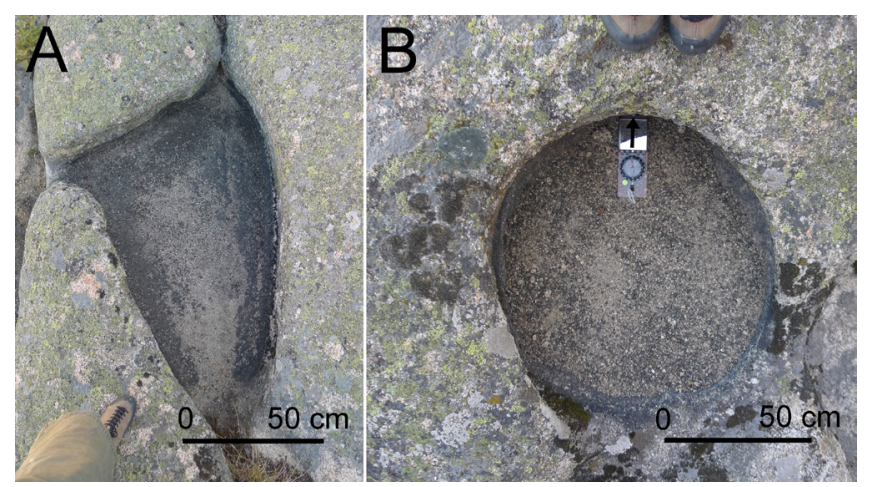

Figura 1 (a) Pila de morfología alargada desarrollada a favor de fracturas, (b) Pila de morfología circular desarrollada a favor de una depresión sobre la superficie de la roca. La Pedriza de Manzanares (Madrid). 
des creando irregularidades. Las biotitas muestran poca disolución aunque sí un grado de oxidación avanzado. Los productos de alteración de la biotita pueden ocupar mayor volumen que la propia biotita, produciendo dilatación y desagregación mineral (Graham y O’Geen, 2010). Por último, los cuarzos también muestran una alteración intensa, aunque comparativamente con los otros minerales es menor. En esencia, la alteración de los cuarzos consiste en una hidratación de la sílice (Dove, 1995; Martini, 2000).

La meteorización física o mecánica que afecta al desarrollo de las pilas incluye básicamente tres mecanismos que pueden actuar de forma independiente: cambios de temperatura por efecto de la insolación, ciclos de hielo-deshielo y cristalización de sales.

La efectividad de los cambios de temperatura dependerá de la insolación y por tanto de la orientación de las rocas (Rice, 1976; Twidale, 1982). En rocas heterogéneas como los granitos, la insolación genera diferencias de temperatura que actúan a escala de los granos minerales de la roca. Estas diferencias de temperatura producen expansiones y contracciones diferenciales de cada grano mineral según su coeficiente específico de dilatación térmica (Twidale y Corbin, 1963; Smith, 1977; Schnepfleitner et al., 2015), que favorece la desagregación mineral de la superficie rocosa (Gómez-Heras et al., 2006; Gómez-Heras et al., 2008). Ishimaru y Yoshikawa (2000) y Hall y André, (2003) consideran al gradiente térmico uno de los principales procesos de la micro-descamación de superficies graníticas.

Las variaciones de temperatura, además de controlar el calentamiento y enfriamiento de la superficie de la roca, regulan los ciclos de hielo y deshielo del agua intersticial presente entre los granos de roca alterada, ayudando a la separación de minerales por crioclastia.

Los cambios de temperatura junto con las modificaciones periódicas de humedad también influyen en la disolución y cristalización de sales (Goudie,
1977; Bradley et al., 1978). Las sales incrementan la solubilidad de los silicatos en periodos húmedos, y cristalizan en periodos secos favoreciendo un proceso de meteorización mixto mecánico y químico.

Además de los procesos químicos y mecánicos citados anteriormente, la presencia de líquenes y musgos pueden jugar un papel importante sobre el control de la alteración superficial de las rocas (Viles y Goudie, 2004). Guando crecen entre las microfisuras de los granos minerales pueden contribuir a la desagregación de la roca por un efecto mixto de tipo físico-químico y biológico (Fry, 1927; Seaward, 1997). Estudios más recientes también señalan a los líquenes no sólo como agentes de meteorización sino como un elemento importante de protección frente a la alteración superficial, contribuyendo a la precipitación de capas ricas en sílice (Chen et al., 2000; Lee y Parsons, 1999). Según Domínguez-Villar (2007) la mayoría de los pilancones inactivos presentan una importante cobertera de líquenes que recubre buena parte de su superficie rocosa, que al sellar la porosidad intergranular contribuyen a preservar ciertas morfologías de las pilas durante periodos de tiempo prolongados. Domínguez-Villar (2007) señala que cuando las paredes de los pilancones no tienen líquenes que preserven la roca frente a la alteración, el efecto de la termoclastia, crioclastia o de precipitación de sales es muy efectivo. En estos casos se produce un retroceso de la pared del pilancón observable incluso en periodos de pocos meses, que se hace patente por la presencia de granos minerales desprendidos de los laterales que se depositan sobre el fondo de la pila.

En general los modelos genéticos publicados que explican el desarrollo de las pilas, indistintamente del clima, coinciden en la existencia e importancia de mecanismos químicos, mecánicos y biológicos, con tasas de meteorización muy diferentes que pueden variar según el peso de cada factor y clima considerado (Smith, 1941; Twidale y Corbin, 1963; Goudie y Migón, 1997). 


\subsection{OBJETIVOS}

El artículo estudia el papel de los ciclos térmicos relacionados con la insolación (Twidale y Corbin, 1963) como un agente más que contribuye al modelado de las paredes de los pilancones. Aunque la crioclastia ha sido considerada por algunos autores como uno de los procesos más significativos en la meteorización física de pilancones (Dahl, 1966; Domínguez-Villar, 2007), en este trabajo se analiza y justifica cómo influye la temperatura en la erosión (concavidad) de las paredes de las pilas en un ambiente climático donde el número de días en los que la temperatura desciende de $0{ }^{\circ} \mathrm{C}$ son muy pocos. En primer lugar se estudia la variabilidad térmica mediante modelos de regresión periódica múltiple, poniendo de manifiesto una influencia diferente de los ciclos diario y anual en función de la orientación de las paredes de las pilas. Finalmente se estudia la asimetría de las paredes de los pilancones teniendo en cuenta las variaciones de temperatura según la insolación recibida (Smith, 1941; Watson y Pye, 1985) entre las caras orientadas al sur y norte. Un modelo matemático de regresión lineal cuantifica la relación existente entre la variabilidad térmica y la alteración de las paredes de los pilancones.

\section{Zona de estudio}

La Pedriza del Manzanares se localiza en las laderas meridionales de la Sierra de Guadarrama, en el Sistema Central Español (Figura 2). Ocupa una superficie de unos $35 \mathrm{~km}^{2}$, entre las zonas de cumbres a unos $2000-2300 \mathrm{~m}$ de altitud y la plataforma de arrasamiento situada a unos 900 metros sobre el nivel del mar. A finales de la orogenia Varisca se produjo la intrusión de cuerpos plutónicos en diversas fases, de las que La Pedriza representa un episodio tardío. Tras un largo periodo de arrasamiento, la orogenia Alpina levantó el Sistema Central actual, reactivando las fracturas variscas y dando origen a una vertiente escalo- nada con relieves grabados (Centeno, 1988). Los leucogranitos peralumínicos (I-type) que forman La Pedriza (Pérez-Soba y Villaseca, 2010), pobres en minerales ferromagnesianos, ha limitado la efectividad de la alteración química, formando un macizo rocoso con muchos afloramientos de roca inalterada y poco meteorizada (Pedraza et al., 2014). Esta característica es la responsable de la ausencia de un espesor significativo de regolito, limitado prácticamente a las zonas de fractura. Por otra parte, la densa red de fracturas ha facilitado la meteorización física y el desarrollo de procesos gravitacionales, dando como resultado un paisaje constituido por una caótica acumulación de bloques (Centeno y García-Rodríguez, 2005). Las rocas filonianas más frecuentes presentes en la zona son de composición aplítica, microdiorítica y de pórfidos graníticos. Los afloramientos graníticos resultantes presentan formas comunes tales como superficies dómicas, tors, piedras caballeras o bien, bolos aislados.

Sobre la superficie de las rocas de La Pedriza es común encontrar formas de meteorización como pilas, acanaladuras, tafoni o agrietamientos poligonales, que fueron clasificadas y sistematizadas en la zona por Pedraza et al., (1989). Algunos trabajos posteriores sobre formas menores en La Pedriza se deben a Domínguez-Villar, (2007), que estudia la morfometría de las pilas y pendientes invertidas, y a García-Rodríguez (2015) y García-Rodríguez et al., (2015a, 2015b) que estudian el desarrollo de formas de alteración expuestas, el control térmico de la meteorización sobre paredes de granito y, los procesos de formación de los agrietamientos poligonales.

En particular, los pilancones que se estudian en este trabajo se desarrollan sobre un leucogranito con tamaño de grano de grueso a medio, formado por cristales de cuarzo, ortosa, microclina, plagioclasa, y biotita subidiomorfa y alotriomorfa, en un porcentaje entre el 2 - 5 \% (García-Rodríguez et al., 2015a). La cementación por óxidos de hierro de las fisuras inter e intracristalinas de estas rocas se debe a la alteración de las biotitas (García-Rodríguez et al., 2015b). Existen minerales 


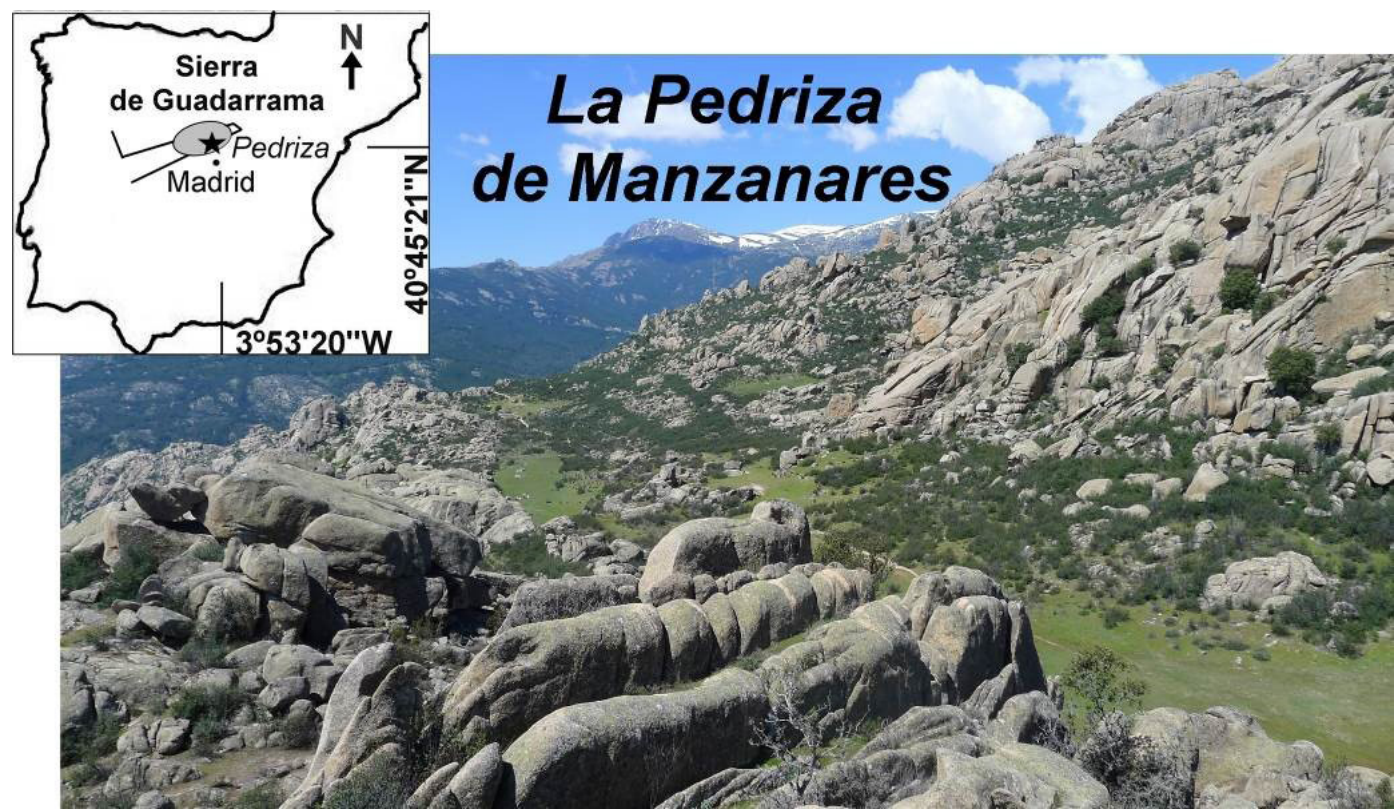

Figura 2 Localización de La Pedriza de Manzanares y paisaje característico de la zona.

secundarios o productos de alteración meteórica, como moscovita, clorita y sericita. En las zonas más superficiales del granito se aprecia una mayor fisuración de los cristales de cuarzo y la cementación de óxidos de hierro es menor en las zonas más internas de la roca. Estas fisuras son de varios milímetros de longitud y presentan aperturas que pueden llegar a los $0.2 \mathrm{~mm}$. En zonas no alteradas estas fisuras pueden estar rellenas con cuarzo.

En la zona, entre altitudes de $800-1200 \mathrm{~m}$, la precipitación media anual es $850 \mathrm{~mm} /$ año con una temperatura media anual de $12{ }^{\circ} \mathrm{C}$. En alturas superiores comprendidas entre $1200-1600 \mathrm{~m}$, la precipitación y temperatura media anual es de $1250 \mathrm{~mm} /$ año y $9{ }^{\circ} \mathrm{C}$ respectivamente. El clima de la zona puede clasificarse como Mediterráneo templado-frío, húmedo (IGME, 1988).

\section{Metodología}

La Pedriza representa un paisaje rocoso (Figura 2) donde la presencia de pilancones representa una forma muy común. Para la consecución de los objetivos propuestos se ha seleccionado una zona que cubre una superficie de cerca de $1 \mathrm{~km}^{2}$ dentro de La Pedriza anterior, entre altitudes de 1100 y $1300 \mathrm{~m}$.

Como el objetivo del trabajo es estudiar el efecto de temperatura sobre la asimetría de las paredes de la pilas, se ha estudiado la franja situada por encima del máximo nivel de agua que puede almacenar la pila tras periodos de lluvia. Esta zona nunca se inunda y la humedad de sus paredes se restringe principalmente a los episodios de lluvia y al tiempo que tarda esa agua en evaporarse de la pared mojada.

La metodología seguida en este trabajo ha sido desarrollada y diseñada a partir de la experiencia y resultados de trabajos previos en la zona (Pedraza et al., 1989; Domínguez-Villar, 2007; García-Rodríguez et al., 2015a). En particular, la secuencia de trabajo ha sido la siguiente:

- Identificación de zonas con abundancia de pilancones en zonas próximas y altitudes similares.

- Selección de un pilancón tipo para monitorizar con sensores $i$-button para registro de temperatura y humedad.

- Inventario de pilas en las que se ha medido la convexidad de sus paredes en las orientaciones norte y sur. 
- Medida de la dureza mediante esclerómetro de la base, paredes y corona de las pilas.

- Análisis matemático y definición de un modelo de los ciclos de temperatura según las orientaciones norte y sur de las paredes de las pilas.

- Estudio de la correlación entre los rangos de temperaturas, concavidad de las paredes de las pilas e índice de esclerometría.

\subsection{I-BUTTONS}

La temperatura y humedad relativa se determinó el empleo de DS1923, de 17.35 mm de diámetro, que registran temperaturas entre $-20{ }^{\circ} \mathrm{C}$ y $+85^{\circ} \mathrm{C}$, y humedad relativa entre 0 a $100 \%$. Los $i$-button Hygrochron miden la humedad del aire a través de un pequeño agujero situado en la superficie del dispositivo. La precisión de los $i$-button es de 0.5 ${ }^{\circ} \mathrm{C}$ para la temperatura y $0.5 \%$ para la humedad relativa.

Se colocaron dos sensores sobre las paredes de las pilas a una altura de 15 centímetros desde el fondo la pila, situados siempre por encima del nivel máximo de agua, coincidiendo también con la zona de mayor concavidad (Figura 3a). El sensor 1/S se colocó en la pared norte de pila (orientado hacia el sur) y el $2 / \mathrm{N}$ en la pared sur de la pila (orientado hacia el norte). Se realizó un registro continuo entre el 1 enero de 2015 y el 31 de diciembre de 2015, registrando medidas de T y HR en intervalos de 10 minutos.

\subsection{MEDIDA DE LA GONGAVIDAD}

Para estudiar la morfología de las paredes de las pilas, se realizaron medidas en una treintena de pilas. La selección de pilas se realizó de forma aleatoria con base en los siguientes criterios: a) que estuvieran sobre superficies planas sin bloques delante que pudieran hacer sombra, b) que si se encontraban sobre grandes bloques, estos no hubieran sido volteados ni basculados, de modo que se garantizara una continuidad en las condiciones de insolación durante todo el tiempo que ha supuesto el proceso de formación de las pilas, c) que el desagüe de las pilas no coincidiera con las direcciones exactas norte y sur, d) que la profundidad de la pila fuera inferior a 50 centímetros para minimizar el efecto de sombra de las propias paredes de la pila, e) que la red de fracturación no fuera un condicionante de la morfología de las paredes, f) que las paredes no estuvieran tapizadas de líquenes que pudieran actuar como un agente de protección ante la alteración y distorsionara el efecto de la temperatura.

$\mathrm{Al}$ medir la curvatura de las paredes, se consideraron valores positivos aquellos donde la pared presentaba concavidad, valor cero si la pared era vertical, y valor negativo si la inclinación de la pared era superior a $90^{\circ}$ en sentido contrario a la concavidad (Figura 3a). Todas las medidas se realizaron en la franja situada por encima del nivel
A

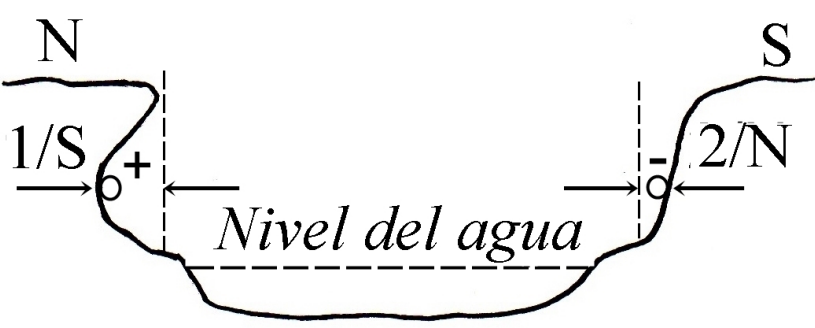

B

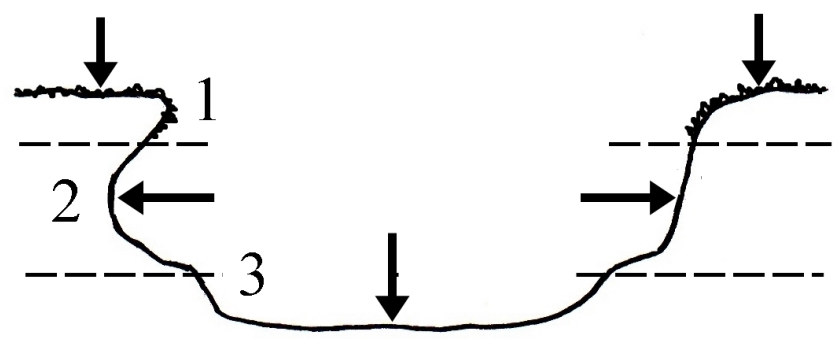

Figura 3 Representación esquemática de una pila tipo orientada según la dirección N-S. (a) Localización con la posición y orientación de los i-button ( $1 / \mathrm{S}$ y $2 / \mathrm{N})$, zonas de medida de la concavidad, posición del nivel máximo de llenado de agua de la pila, (b) Posiciones de medida del índice de esclerometría (flechas negras). (1) Zona superior colonizada por líquenes, (2) zona activa intermedia, (3) zona comprendida entre la base de la pila y el nivel máximo de llenado de agua. 
máximo del agua de la pila. En cada pila y según las orientaciones sur y norte, se realizaron un total de 10 medidas sobre una franja de unos $10 \mathrm{~cm}$, para trabajar con los valores medios.

\subsection{DUREZA}

Se realizaron medidas del índice de esclerometría con martillo Schmidt (Modelo GeoHammer DRG 14GO200L), conforme a los métodos empleados en estudios geomorfológicos por autores previos (Goudie, 2006) así como en otros trabajos recientes desarrollados en los granitoides de La Pedriza (García-Rodríguez et al., 2015a; García-Rodríguez y Fernández-Escalante, 2016).

Las medidas se tomaron en pilancones secos y en diferentes zonas; en la corona superficial externa, en la zona central del fondo de la pila, y en las paredes laterales orientadas al sur y norte (Figura 3b). Para descartar ruidos en el análisis estadístico, durante las medidas se evitaron zonas con irregularidades anómalas, como por ejemplo con protuberancias debidas a fenocristales o áreas con descamaciones muy evidentes. En la corona se tomaron un mínimo de 30 medidas y hasta las necesarias para cubrir todo el perímetro dependiendo del tamaño de la pila. Las medidas de cada cara se tomaron utilizando la línea de agua de máximo llenado como patrón de guía de las medidas, tomándose siempre por encima y sobre la zona fósil de la pila. En el fondo de cada las pilas se realizaron también 20 medidas. El número total de datos para su análisis estadístico fue de 798 medidas.

\subsection{ANÁLISIS ESTADÍSTICO}

El estudio de la temperatura se abordó mediante regresiones periódicas con dos ciclos: anual y diario, la interacción de ambos y la humedad. El modelo de regresión obtenido es el siguiente:

$$
\hat{T}_{t_{1}, t_{2}}=\beta_{0}+\sum_{i=1}^{2} \beta_{i} \sin \left(\alpha_{i}\right)+\beta_{3} \prod_{i=1}^{2} \sin \left(\alpha_{i}\right)+\beta_{4} H ; \quad \alpha_{i}=\frac{2 \pi t_{i}}{P_{i}}+\phi_{i}
$$

Donde $t_{1}$ y $t_{2}$ representan el día y la hora respectivamente, $\hat{T}_{t_{1}, t_{2}}$ es la predicción de temperatura, $P_{i}$ y $\phi_{i}$ son el periodo (en horas y días; 24 y 365, res- pectivamente) y la fase del ciclo i-ésimo y $H$ es la humedad.

La fase de los ciclos se calcula a partir del correspondiente modelo de regresión incluyendo junto con el seno la función coseno como factor, sin considerar la fase. Si $\gamma_{1}$ y $\gamma_{2}$ son los parámetros asociados al seno y al coseno en dicho modelo, la fase del ciclo es $\phi=\arctan \left(\gamma_{1} / \gamma_{2}\right)$, dependiendo el cuadrante del ángulo del signo de los dos parámetros. La comparación de los parámetros entre los diferentes sensores se realizó mediante partial F-tests.

Las comparaciones del rango de temperaturas y la concavidad en las diferentes orientaciones de los sensores se realizaron mediante una t de Student. La dependencia de la concavidad con respecto al rango de temperatura se analizó con regresión lineal. El índice de esclerometría entre el fondo y la corona y entre la cara orientada al Norte y la orientada al Sur se comparó mediante una t de Student. En todos los contrastes realizados se adoptó un nivel de significación $a=0.05$. Todos los análisis estadísticos se realizaron con el software estadístico STATA v. 9.0.

\section{Resultados}

\subsection{ZONIFICACIÓN DE LAS PAREDES DE LAS PILAS}

El estudio realizado ha permitido constatar que, en general, la presencia de líquenes en las pilas de la Pedriza contribuye a proteger la roca frente a la alteración, conclusión que ya era anunciada por Domínguez Villar (2007). Este hecho se hace patente en la mayoría de pilas estudiadas independientemente de su forma, diámetro y profundidad. Por ejemplo, la distribución y continuidad de una superficie tapizada por líquenes ya delimita la forma exterior de la pilas en su estado inicial, cuando empiezan a formarse sobre superficies planas a favor de pequeñas depresiones (Figura 4). Cuando las pilas evolucionan y se desarrollan en la vertical aumentando su profundidad (Figura 5), se ha observado que los líquenes, además de cubrir la corona exterior tienden a colonizar la parte supe- 
rior de las paredes representando una protección frente a la alteración. El efecto protector de los líquenes en la parte superior de las paredes es tanto mayor cuanto más profunda es la pila.

$\mathrm{El}$ análisis de morfométrico de las paredes de las pilas estudiadas ha denotado una clara asimetría dependiendo de su orientación sur o norte, con una concavidad más pronunciada en las paredes de las pilas orientadas al sur (Figura 6). Los valores medios y las desviaciones estándar obtenidos de cada uno de los cuatro parámetros medidos fueron:

- Tamaño del eje de la pila en la dirección N-S $(\mathrm{mm}): 877.3 \pm 78.6$

- Profundidad máxima de la pila (mm): 311.8 $\pm 42.2$

- Erosión pared norte, orientación sur $(\mathrm{mm})$ : $63.5 \pm 10.1$

- Erosión pared sur, orientación norte (mm): 6.9 $\pm 3.6$

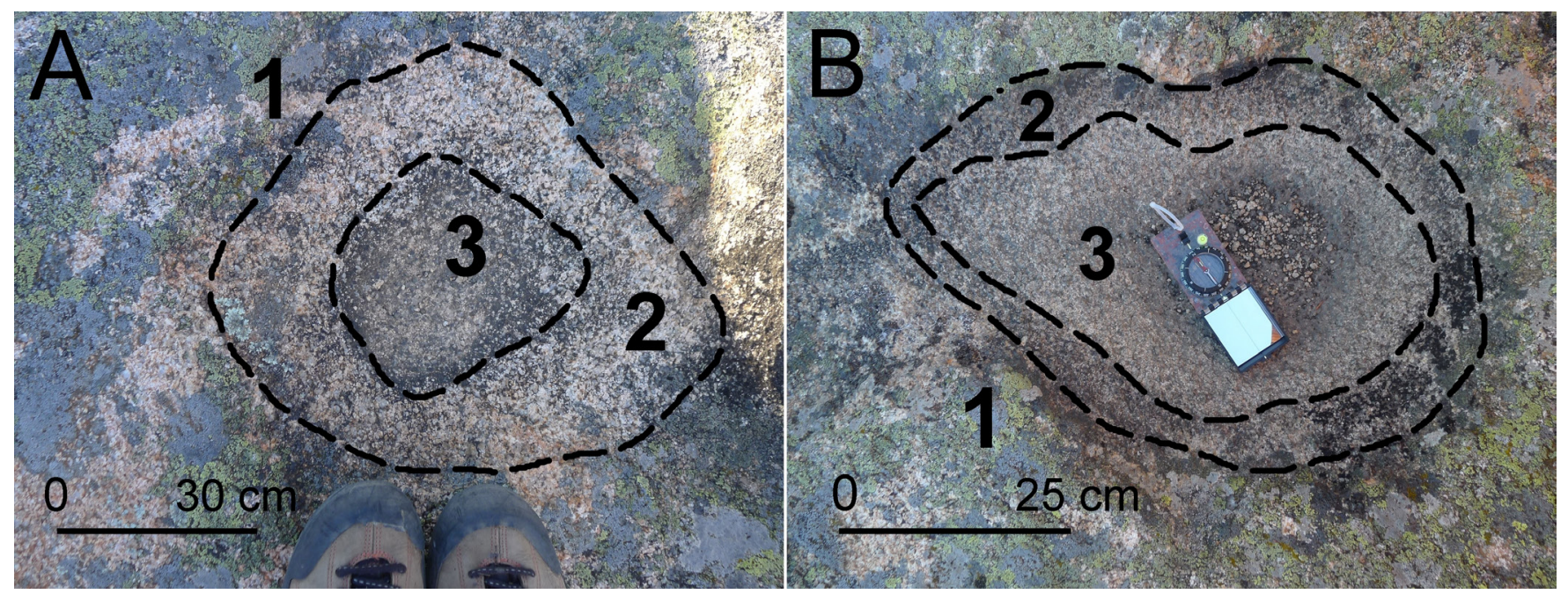

Figura 4 Ejemplos de pilas incipientes desarrolladas sobre superficies planas. (a) Pila incipiente de aspecto circular de apenas $2 \mathrm{~cm}$ de profundidad, (b) Pila de forma elongada con una profundidad máxima de $5 \mathrm{~cm}$. (1) Superficie de la roca colonizada por líquenes, (2) franja intermedia entre el nivel habitual de máximo llenado de agua borde exterior de la pila, (3) zona máxima habitual de llenado de agua.

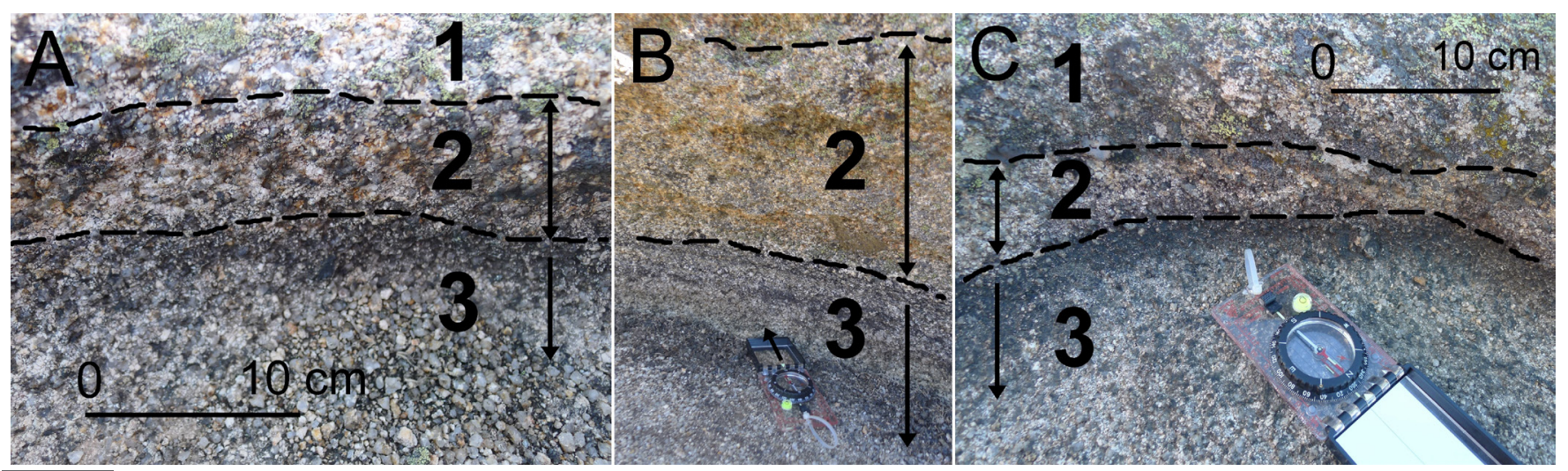

Figura 5 (a), (b) y (c) Ejemplos de tres paredes internas de pilas con indicación de tres zonas bien definidas, (1) Zona superior del borde de la pila con una cobertera de líquenes continua donde el granito presenta un bajo grado de desagregación, (2) zona intermedia entre el nivel máximo de agua en la pila y la zona colonizada por líquenes. Se trata de la zona permanentemente aérea que presenta mayor alteración, (3) zona comprendida entre el nivel máximo de agua en la pila y fondo. 
El estudio de la temperatura sobre la franja activa de las paredes de las pilas situadas justo por encima del máximo nivel de llenado de agua, ha permitido establecer relaciones entre la concavidad de las paredes y los ciclos de temperatura en las distintas orientaciones, como un factor más determinante de estas formas asimétricas.

\subsection{AJUSTE DE LA TEMPERATURA MEDIANTE UN MODELO DE REGRESIÓN PERIÓDICA MÚLTIPLE}

Las temperaturas máximas anuales en las orientaciones sur y norte, se tienen en los meses de junio y julio alcanzando los mismos valores, siendo enero el mes dónde la diferencia de $\mathrm{T}^{\mathrm{a}}$ entre ambos sensores es mayor (Tabla 1). Las $\mathrm{T}^{\mathrm{a}}$ mínimas, tienen idénticos valores en los meses desde abril a diciembre, siendo febrero donde la diferencia de temperatura es mayor entre ambas orientaciones. Los datos de la Tabla 1 demuestran que el ángulo de incidencia de la radiación solar en la cara sur es inferior durante el solsticio de verano que durante

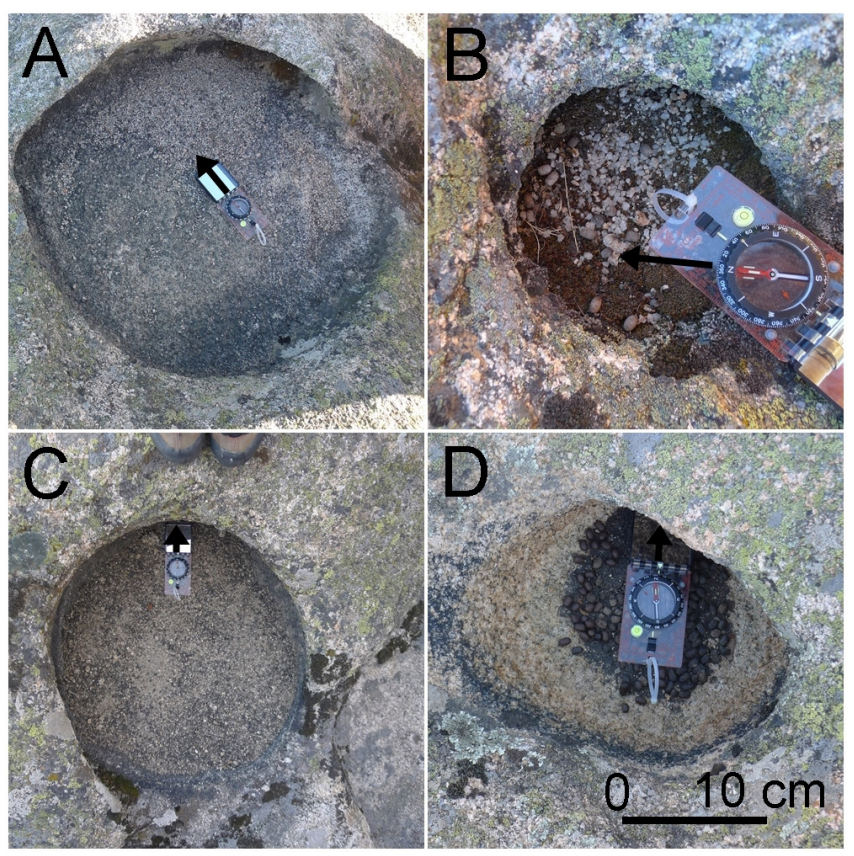

Figura 6 (a), (b), (c) y (d) Ejemplos de pilas que denotan asimetría entre las orientaciones sur y norte de sus paredes. En todos los casos la máxima concavidad se localiza en las paredes orientadas al sur. el equinoccio de otoño debido a la altura del sol sobre el horizonte.

Los ciclos diarios están impuestos por el ciclo anual. En algunos casos las series de medidas pueden presentar ciertas complicaciones (en relación con valores de $\mathrm{T}^{\mathrm{a}}$ máximos) debido a la propia geometría de las paredes de las pilas y profundidad de las mismas. En relación con los ciclos diarios se pueden producir fluctuaciones de temperatura en espacio de tiempo corto debido al efecto del viento o a la presencia de nubosidad.

La temperatura se ajustó en función de tiempo, teniendo en cuenta el día y la hora, aplicando un modelo de regresión periódica múltiple. Para cada sensor la temperatura se ha ajustado a un ciclo diario $\left(a_{1}\right)$ con una fase de 3.625 radianes (13.8 horas), a un ciclo anual $\left(a_{2}\right)$ con una fase de 4.295 radianes (249.5 días), a la humedad $(H)$, y a la interacción de ambos ciclos. Los cuatro factores fueron significativos en los 2 sensores (1/S y $3 / \mathrm{N})$, dando lugar a los siguientes modelos de regresión:

$\hat{T}_{S \mid t_{1}, t_{2}, H}=25.75+7.37 \sin \left(\alpha_{1}\right)+3.76 \sin \left(\alpha_{2}\right)+0.26 \sin \left(\alpha_{1}\right) \sin \left(\alpha_{2}\right)-0.17 H, \quad r^{2}=0.81$

$\hat{T}_{N \mid t_{1}, t_{2}, H}=22.44+8.79 \sin \left(\alpha_{1}\right)+2.97 \sin \left(\alpha_{2}\right)+1.65 \sin \left(\alpha_{1}\right) \sin \left(\alpha_{2}\right)-0.13 H, \quad r^{2}=0.86$

Donde $\hat{T}_{\left.K\right|_{1}, t_{2}, H}$ es la predicción de Temperatura para el sensor con orientación $K$, en la hora $t$, del día $t_{2}$ con Humedad $=H$.

La interacción entre los dos ciclos significa que el efecto de la hora sobre la temperatura depende de la época del año y viceversa. Al comparar los dos sensores, se ha visto que en todas las variables del modelo hay diferencias significativas, es decir, el efecto de la humedad, los ciclos y su interacción sobre la temperatura medida por el sensor, difiere en función de la posición del sensor $(p<0.0001$ para todos los factores).

Los ajustes del modelo de regresión correspondientes a sensores $1 / \mathrm{S}$ y $2 / \mathrm{N}$ presentan una elevada variabilidad en cuanto al rango de temperaturas. En las figuras 7 y 8 se presentan los resultados del modelo para los dos sensores con la predicción de la temperatura para todos los días del año (figuras 7a y 8a) así como una ampliación de la predicción de la temperatura correspondiente al periodo 
comprendido entre los días 8 y 28 de febrero. La capacidad predictiva del modelo se aprecia en las figuras con ampliación, donde el ajuste coincide casi perfectamente con los registros de temperatura medidos. En las figuras con ampliación (figuras 7 b y $8 b$ ) también se puede ver que cada día existen momentos puntuales en los que se alcanza valores máximos de temperatura relacionados con la insolación directa.
Los resultados del modelo indican una variabilidad de los rangos de temperatura tanto el ciclo diario como anual en los dos sensores. Como cabe esperar las diferencias de los rangos de temperatura diarios de los sensores son mayores en los meses de invierno, disminuyendo en la estación estival. En el mecanismo de calentamiento de la roca influyen dos procesos (Gómez-Heras et al., 2008): el calentamiento por la insolación directa y las va-

Tabla 1. Temperaturas máximas y mínimas mensuales $\left({ }^{\circ} \mathrm{C}\right)$ registradas en los sensores $1 / \mathrm{S}$ y $2 / \mathrm{N}$.

\begin{tabular}{|c|c|c|c|c|c|c|c|c|c|c|c|c|}
\hline & Ene. & Feb. & Mar. & Abr. & May. & Jun. & Jul. & Ago. & Sep. & Oct. & Nov. & Dic. \\
\hline$(1 / \mathrm{S})$ & $36 /-2$ & $22 /-2$ & $38 / 0$ & $35 / 7$ & $42 / 8$ & $40 / 10$ & $47 / 17$ & $46 / 13$ & $41 / 8$ & $34 / 7$ & $38 / 1$ & $38 / 4$ \\
\hline$(2 / \mathrm{N})$ & $12 /-3$ & $12 /-5$ & $22 /-1$ & $31 / 7$ & $41 / 8$ & $40 / 10$ & $47 / 17$ & $41 / 13$ & $37 / 8$ & $25 / 7$ & $25 / 1$ & $15 / 3$ \\
\hline
\end{tabular}

A
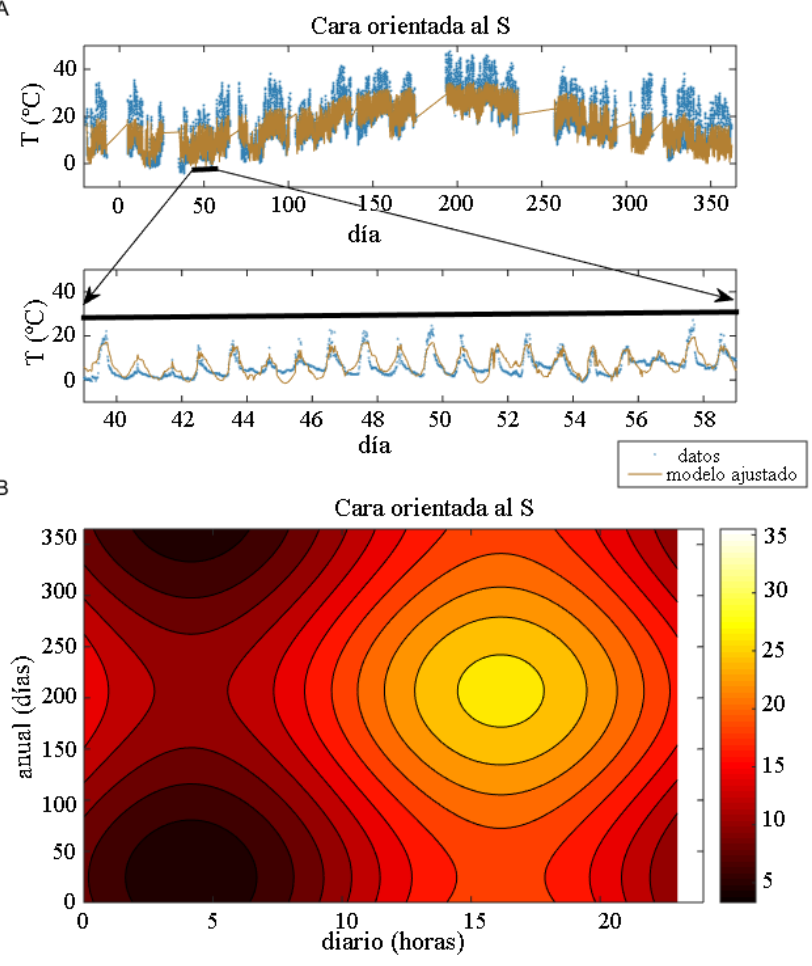

Figura 7 Datos reales y modelos de regresión obtenidos de la cara orientada al Sur. A) Datos de temperatura obtenidos a lo largo de un año (puntos azules) y el ajuste obtenido por medio de una regresión periódica (líneas marrones). Debajo de cada gráfica se ha ampliado la región correspondiente a los días 39 a 59. B) Predicciones de temperatura en función de los ciclos anual y diario para una humedad constante del $62 \%$, correspondiente a la media anual. Las isóclinas muestran de manera clara las interacciones entre los dos ciclos. La escala de color muestra la temperatura en ${ }^{\circ} \mathrm{C}$.

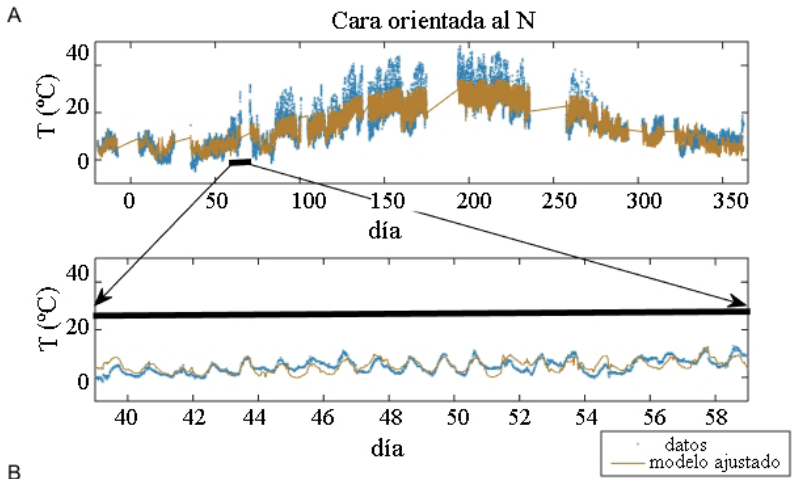

B

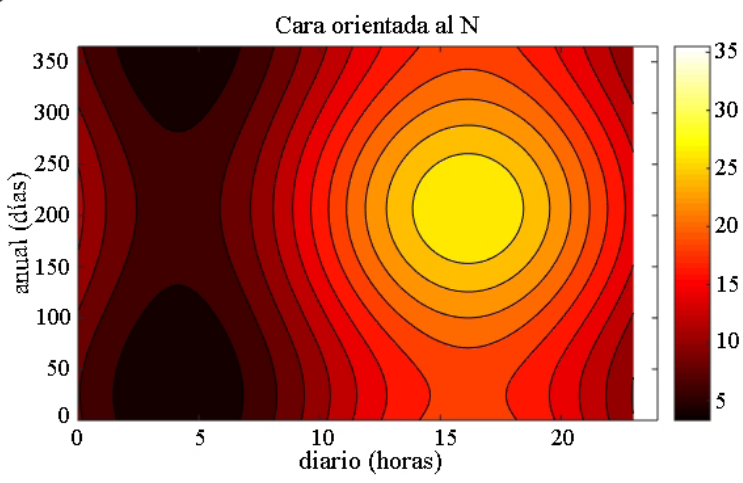

Figura 8 Datos reales y modelos de regresión obtenidos de la cara orientada al Norte. A) Datos de temperatura obtenidos a lo largo de un año (puntos azules) y el ajuste obtenido por medio de una regresión periódica (líneas marrones). Debajo de cada gráfica se ha ampliado la región correspondiente a los días 39 a 59. B) Predicciones de temperatura en función de los ciclos anual y diario para una humedad constante del $62 \%$, correspondiente a la media anual. Las isóclinas muestran de manera clara las interacciones entre los dos ciclos. La escala de color muestra la temperatura en ${ }^{\circ} \mathrm{C}$. 
riaciones de temperatura controladas por procesos convectivos puros. Generalmente los valores máximos alcanzados tienen relación con la insolación. Los procesos convectivos contribuyen homogenizando la temperatura de todas las paredes de la pila por igual, estando especialmente presentes en los periodos de tiempo en los que se alcanzan las temperaturas mínimas y medias. Cuando el calentamiento de la roca se debe a la influencia directa de la radiación solar, los gradientes de temperatura son elevados y tienen una duración de tiempo breve. Estos gradientes de temperatura (Smith et al., 2011) están controlados por el tiempo e intensidad del proceso de calentamiento, creando ciclos en los que la roca se calienta y enfría muy rápidamente.

La comparación de las predicciones de temperaturas de los sensores $1 / \mathrm{S}$ y $2 / \mathrm{N}$ (Figuras 7 y 8 ) indican una variabilidad importante de los rangos de $\mathrm{T}^{\mathrm{a}}$ de los datos diarios, tanto para las medidas reales como para los datos simulados:

- Las temperaturas máximas registradas en las paredes de las pilas con orientación sur están afectadas durante los ciclos, anual y diario, por el efecto de la insolación directa. Las temperaturas mínimas en ambos ciclos (anual y diario) guardan relación con procesos convectivos.

- Durante los meses centrales del año (primavera y verano), el calentamiento de las paredes orientadas al norte tienen el mismo comportamiento general que en las orientadas al sur. Durante los meses de invierno las temperaturas máximas disminuyen respecto a la orientación sur, siendo mínima en la cara orientada al norte.

\subsection{RELAGIÓN GONGAVIDAD - TEMPERATURAS}

El análisis de la relación entre la concavidad de las paredes de las pilas en diferentes orientaciones con la temperatura en cada orientación, se ha seguido la siguiente línea argumental: a) comparar el rango de temperaturas de los dos sensores teniendo en cuenta su orientación, b) relacionar la profundidad de la concavidad con su orientación y, c) relacionar la profundidad de la concavidad con la temperatura.

a) Se han analizado los siguientes valores diarios relativos a la temperatura $(\mathrm{T})$ : media, máximo, mínimo y rango (máximo - mínimo). Dichos valores se han comparado entre las dos caras del pilancón (N y S). En todas las variables estudiadas, exceptuando la temperatura mínima $(p=0.32, \mathrm{t}$-student) se han encontrado diferencias significativas entre ambas caras. De este modo la cara orientada al sur presenta en promedio valores mayores de temperatura media diaria $(p=0.02$, t-student $)$, temperatura máxima diaria $\left(p=2.0 \mathrm{e}^{-10}, \mathrm{t}\right.$-student $)$ y rango de temperatura diario $(p=0.0$, t-student $)$ (Figura 9).

b) La comparación entre la concavidad de las dos caras de los pilancones arrojó diferencias significativas ( $p=1.7 \mathrm{e}^{-5}$, t-student) entre la concavidad de la cara orientada al sur, que es la que mayor concavidad presenta (Figura 9). El sensor 1/S presenta, en promedio, un mayor rango de variación de temperatura, frente al sensor $2 / \mathrm{N}$ que es el que menor variación promedio de temperatura sufre.

c) Una regresión simple tomando como variable de interés la erosión (concavidad) y como variable explicativa el rango de temperatura, da el modelo, $\hat{C}=-628.04+40.6 \cdot T_{\text {rango }}\left(R^{2}=44.4 \%\right)$ que indica que por cada unidad que aumenta el promedio anual del rango de temperatura se incrementa en 40.6 la concavidad $(p<0.00001)$. $\mathrm{Al}$ comparar los registros de temperatura de las orientaciones sur y norte con la asimetría observada en la concavidad de las paredes de las pilas, se confirma la importancia que tiene la radiación solar en breves periodos de tiempo (Gómez-Heras et al., 2008; García-Rodríguez et al., 2015a). Como el efecto de la meteorización por cambios de temperatura guarda relación con el número de ciclos de cambios de temperatura, los resultados de esta correlación y modelo indican que en el ambiente climático en el que se desarrolla el trabajo, el efecto de la amplitud del rango de temperatura sobre la zona fósil de las pilas puede considerarse como un factor relevante responsable de la alteración de las paredes aumentando su concavidad. 


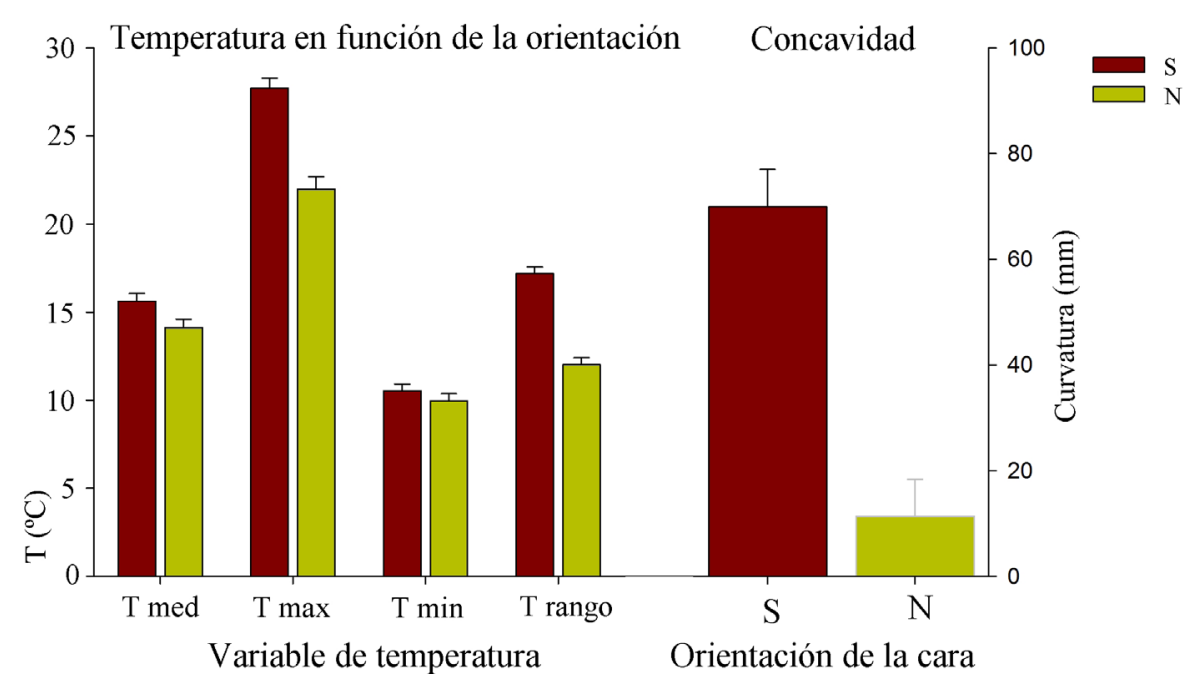

Figura 9 Valores medios de las variables de temperatura (media, máximo, mínimo y rango diarios) y de la curvatura de las caras orientadas al norte y al sur.

\subsection{DUREZA}

Existe un gran número de factores que se debe tener en consideración al estudiar e interpretar resultados referentes a la dureza de rocas que se encuentran sometidas a un proceso de meteorización activo. Por ejemplo, algunos factores a tener en consideración son el contenido en agua, condiciones ambientales puntuales, como por ejemplo precipitaciones recientes, la presencia de superficies endurecidas o escamas de alteración (Dorn et al., 2013), tamaño de los minerales que componen la roca, o la presencia de microorganismos tales como líquenes. Los líquenes pueden contribuir a la cementación de la roca, depositando solutos como por ejemplo de sílice (Chen et al., 2000). En este mismo sentido Ascaso y Wierchos (1994) ya evidenciaban que algunos líquenes muy frecuentes en rocas graníticas (p.ej. Parmelia conspersa) pueden incorporar en sus talos liquénicos trozos de minerales de la roca sobre la que se encuentran. Si la cobertera de líquenes es amplia, este proceso puede contribuir a sellar la porosidad intergranular proporcionado una pátina protectora que estabiliza la superficie rocosa retardando la alteración. Los resultados de dureza obtenidos en cada cara pueden verse en la (Tabla 2). Al comparar la du- reza de la corona de las pilas con su fondo, se obtiene un $p$-valor $=0.0$ (t-student), denotando una clara diferencia entre la dureza de la corona y la del fondo. Los valores de dureza de la cara norte y la sur dan un $p$-valor $=0.94$ (t-student), indicando que no se observan diferencias de dureza entre ambas caras. Este resultado contribuye a justificar que, si bien el estudio se realizó sobre superficies con características homogéneas en cuanto a su dureza, el proceso de desagregación granular es más rápido en la cara orientada al Sur, tal y como denota la relación descrita entre concavidad y temperatura de las caras Sur y Norte. Las pilas estudiadas han puesto de manifiesto un mayor desarrollo lateral, a favor de las paredes, que en la vertical en profundidad, consistente con los datos de dureza.

El índice de esclerometría medido en las coronas y paredes de las pilas es ligeramente menor que el obtenido sobre el fondo, que presenta un superficie más estable y sin líquenes.

Si bien la presencia de líquenes no proporciona dureza a la corona y paredes de las pilas en un clima Mediterráneo templado-frío, se ha comprobado que su presencia contribuye proporcionando compacidad a una superficie que sin su existencia tendría un grado de desagregación mayor. 
Tabla 2. Media, desviación típica y tamaño de las muestras de dureza de cada una de las caras.

\begin{tabular}{|c|c|c|c|c|}
\hline & Corona & Fondo & Norte & Sur \\
\hline N & 367 & 141 & 143 & 151 \\
\hline Media & 36.77 & 42.14 & 37.7 & 37.64 \\
\hline Desviación típica & 6.36 & 4.7 & 7 & 6.79 \\
\hline
\end{tabular}

Aunque son muchos los factores que influyen en la meteorización de las pilas (Blum y Stillings, 1995; Viles y Goudie, 2004; Domínguez-Villar, 2007; Domínguez-Villar, 2008), los resultados que se presentan en este trabajo, referidos a un clima donde los cambios de temperatura son muy grandes y se producen con mucha rapidez, han demostrado la importancia de la orientación de las paredes de las pilas en el proceso de meteorización dando lugar a geometrías muy bien definidas y diferentes entre las orientaciones sur y norte. Trabajos previos en este sentido (Twidale y Corbin, 1963; Smith, 1977; Hall y André, 2003) ya apuntaban la importancia de la temperatura como agente de modelado de formas graníticas.

\section{Conclusiones}

El tratamiento estadístico de medidas de alta resolución de temperatura y humedad han permitido definir un modelo para la simulación de la temperatura ciclo anual y diario en las orientaciones norte y sur de las caras de las pilas.

La combinación del modelo de temperaturas con el tratamiento estadístico de las medidas de concavidad de las paredes de las pilas ha permitido identificar una clara correlación entre los rangos de temperatura registrados en las orientaciones norte y sur con el grado de concavidad.

Los ciclos diarios de temperatura están controlados por los ciclos anuales. La modelización de las variaciones de temperatura de las caras con orientación sur y norte ha demostrado que los mayores rangos corresponden a las orientaciones sur. La curva de los ciclos de temperatura que ajusta el modelo suaviza las temperaturas máximas y mínimas puntuales, asimilando el régimen térmico a un ambiente convectivo. No obstante el modelo también es coherente con las diferencias entre las temperaturas máximas y mínimas registradas a nivel diario.

El modelo y resultados obtenidos confirman el papel de la temperatura como un factor de especial relevancia en el proceso de meteorización de las pilas graníticas en clima mediterráneo dónde la temperatura desciende de $0^{\circ} \mathrm{C}$ sólo unos pocos días al año.

\section{Agradecimientos}

Los autores agradecen al proyecto MINECO (CGL2013-40851-P) "Diversidad, bioindicación y biorremediación de protistas en ecosistemas protegidos de paisaje granítico. Hacia estrategias de conservación de especies" (Microepics).

\section{Referencias}

Alexandrowicz, Z., 1989, Evolution of weathering pits on sandstone tors in the Polish Carpatians: Zeitschrift für Geomorphologie, 33, 275-289.

Ascaso, C., Wierchos, J., 1994, Structural aspects of the lichen-rock interface using backscattered electron imaging: Botanica Acta, 107, 252-256.

Bradley, W.C., Hutton, J.T., Twidale, G.R., 1978, Role of salts in the development of granitic tafoni, South Australia: The Journal of Geology, 86, 647-654, DOI: 10.1086/649730.

Blum, A.E., Stillings, L.L., 1995, Feldespar dissolution kinetics, in Chemical weathering rates of silicate minerals, en White, A.F., Brantley, S.L. (ed.), Reviews in Mineralogy, 31: Washington, Mineralogical Society of America, 291-351. 
Brady, P.V., Walther, J.V., 1990, Kinetics of quartz dissolution at low temperatures: Chemical Geology, 82, 253-264, DOI: 10.1016/0009-2541(90)90084-K.

Branner, J.C., 1913, The fluting and pitting of granites in the tropics: American Philosophy Society Proceedings, 52, 163-174.

Centeno, J.D., 1988, Morfología granítica de un sector del Guadarrama Occidental: Madrid, Universidad Complutense de Madrid, tesis doctoral no 262/88, $321 \mathrm{p}$.

Centeno, J.D., García-Rodríguez, M., 2005, El papel de los procesos gravitacionales en los relieves graníticos: el derrumbe de Peña Sirio (Pedriza de Manzanares, Madrid): Tecnologi@y Desarrollo,3, 1-18.

Chen, J., Blum, H-P., Beyer, L., 2000, Weathering of rocks induced by lichen colonization, a review: Catena, 39, 121-146, DOI: 10.1016/ S0341-8162(99)00085-5.

Dahl, R., 1966, Block fields, Weathering pits and tor-like forms in the Narvik Mountains, Nordland, Norway: Geografiska Annaler, 48a, 55-85.

Domínguez-Villar, D., 2007, Análisis morfométrico de pilancones consideraciones genéticas, evolutivas y paleoambientales: Madrid, Universidad Complutense de Madrid, tesis doctoral, $336 \mathrm{p}$.

Domínguez-Villar, D., Jennings, C.E., 2008, Multi-phase evolution of gnammas (weathering pits) in a Holocene deglacial granite landscape, Minnesota (USA): Earth Surface Processes and Landforms, 33, 165177, DOI: 10.1002/esp.1532.

Domínguez-Villar, D., Arteaga, C., GarcíaGiménez, R., Smith, E.A., de Pedraza, J., 2008, Diurnal and seasonal water variations of temperature, $\mathrm{pH}$, redox potential and conductivity in gnammas (weathering pits): Implications for chemical weathering: Catena, 72, 37-48, DOI: 10.1016/j. catena.2007.03.018.

Domínguez-Villar, D., Razola, L., Carrasco, R., Jennings, C.E., de Pedraza, J., 2009,
Weathering phases recorded as gnammas developed since last glaciation at Serra da Estrela, Portugal: Quaternary Research, 72, 218-228.

Dorn, R.I., 1995, Digital processing of back-scatter electron imagery: A microscopic approach to quantifying chemical weathering: Geological Society of America Bulletin, 107, 725-741.

Dorn, R.I., Gordon, S.J., Allen, C.D., Cerveny, N., Dixon, J.C., Groom, K.M., Hall, K., Harrison, E., Mol, L., Paradise, T.R., Sumner, P., Thompson, T., Turkington, A.V., 2013, The role of fieldwork in rock decay research: Case studies from the fringe: Geomorphology, 200, 59-74.

Dove, P.M., 1995, Kinetic and thermodynamic controls on silica reactivity, en White, A.F., Brantley, S.L. (ed.), Chemical weathering rates of silicate minerals: Washington, Mineralogical Society of America, Reviews in Mineralogy, 31, 235-290.

Fry, E.J., 1927, The mechanical action of crustaceous lichens on substrata of shale, schist, gneiss limestone and obsidian: Annals of Botany, 41, 437-460.

García-Rodríguez, M., 2015, Erosión y exhumación de bloques graníticos en La Pedriza del Manzanares (España). Evolución histórica a partir de dataciones relativas: Revista Mexicana de Ciencias Geológicas, 32(3), 492-500.

García-Rodríguez, M., Fernández-Escalante, A.E., 2016, Geo-Climbing and Environmental Education: the Value of La Pedriza Granite Massif in the Sierra de Guadarrama National Park, Spain: Geoheritage, DOI: 10.1007/ s12371-016-0187-y.

García-Rodríguez, M., García-Rodríguez, M., Gómez-Heras, M., Fort, R., Álvarez de Buergo M., 2015a, Control térmico de la meteorización de superficies endurecidas en rocas graníticas (La Pedriza de Manzanares, España): Boletín de la Sociedad Geológica Mexicana, 67(3), 533-544. 
García-Rodríguez, M., Gómez-Heras, M., Álvarez de Buergo, M., Fort, R., Aroztegui, J., 2015b, Polygonal cracking associated to vertical and subvertical fracture surfaces in granite (La Pedriza del Manzanares, Spain): considerations for a morphological classification: Journal of Iberian Geology, 41(3), 365-383, DOI: $\quad 10.5209 / \mathrm{rev}_{-}$ JIGE.2015.v41.n3.48860.

Gómez-Heras, M., Smith, B.J., Fort, R., 2006, Surface temperature differences between minerals in crystalline rocks: Implications for granular disaggregation of granites through thermal fatigue: Geomorphology, 78(3-4), 236-249, DOI: $10.1016 / \mathrm{j}$. geomorph.2005.12.013.

Gómez-Heras, M., Smith, B.J., Fort, R., 2008, Influence of surface heterogeneities of building granite on its thermal response and its potential for the generation of thermoclasty: Environmental Geology, 56(3-4), 547-560, DOI: $10.1007 /$ s00254-008-1356-3.

Goudie, A.S., 1977, Sodium sulphate weathering and the disintegration of MohenjoDaro, Pakistan: Earth Surface Processes and Landforms, 2(1), 75-86, DOI: $10.1002 \% 2$ fesp.3290020108.

Goudie, A.S., 2006, The Schmidt Hammer in geomorphological research: Progress in Physical Geography, 30(6), 703-718.

Goudie, A.S., Migón, P., 1997, Weathering pits in the Spitzkoppe area, Central Namib Desert: Zeitschrift für Geomorphologie, 41(4), 417-444.

Graham, R.C., O’Geen, A.T., 2010, Soil mineralogy trends in California landscapes: Geoderma, 154, 418-437, DOI: 10.1016/j. geoderma.2009.05.018.

Gutiérrez Elorza, M., Ibáñez, M.J., 1979, Las "gnammas" de la región de Alcañiz: Estudios Geológicos, 35, 193-198.

Hall, K., André, M.F., 2003, Rock thermal data at the grain scale: applicability to granular disintegration in cold environments: Earth
Surface Processes and Landforms, 28, 823836, DOI: 101002/esp.494.

Hall, A.M., Phillips, W.M., 2006, Weathering pits as indicators of the relative age of granite surfaces in the Cairngorm Mountains, Scotland: Geografiska Annaler, 88A, 135-150, DOI: $10.1111 / \mathrm{j} .0435-3676.2006 .00290 . x$.

Instituto Geológico y Minero de España (IGME), 1988, Atlas Geocientífico del Medio Natural de la Comunidad de Madrid: Madrid, IGME y Comunidad de Madrid, 83 p.

Ishimaru, S., Yoshikawa, K., 2000, The weathering of granodiorite porphyry in the Thiel Mountains, inland Antartica: Geografiska Annaler, 82A, 45-57.

Lee, M.R., Parsons, I., 1999, Biomechanical and biochemical weathering of lichen-encrusted granite: textural controls on organic-mineral interactions and deposition of silica-rich layers: Chemical Geology, 161, 385-397, DOI: 10.1016/S0009-2541(99)00117-5.

Matthes, F.E., 1930, Geologic history of the Yosemite Valley: U.S. Geological Survey Professional Paper 160.

Maclaren, M., 1912, Notes on desert-water in Western Australia. "Gnamma" holes and "night-wells": Geological Magazine, 9, 301-304.

Martini, J.E.J., 2000, Dissolution of quartz and silicate minerals, en Klimchouk, A.B., Ford, D.C., Palmer, A.N., Dreybrodt, W. (ed.), Speleogenesis, Evolution of karst aquifers: Hunstville, National Speleological Society, 171-174, DOI: 10.1016/ S0022-1694(00)00341-3.

Mol, L., Viles, A., 2012, The role of rock surface hardness and internal moisture in tafoni development in sandstone: Earth Surface Processes and Landforms, 37, 301-314, DOI: 10.1002/esp.2252.

Pedraza, J., Sanz, M.A., Martín, A., 1989, Formas graníticas de la Pedriza: Madrid, Agencia de Medio Ambiente Comunidad de Madrid, $205 \mathrm{p}$. 
Pedraza, J., Carrasco, M.R., Domínguez-Villar, D., 2014, Geomorphology of La Pedriza Granitic Massif, Guadarrama Range, en Gutiérrez, F., Gutiérrez, M., (ed.), Landscapes and Landforms of Spain: Netherlands, Springer, 71-81.

Pérez-Soba, C., Villaseca, C., 2010, Petrogenesis of highly fractionated I-type peraluminous granites: La Pedriza pluton (Spanish Central System): Geologica Acta, 8, 131-149.

Rice, A., 1976, Insolation warmed over: Geology, 4, 61-62, DOI: 10.1344/105.000001527.

Seaward, M.R.D., 1997, Mayor impact made by lichens in biodeterioration processes: International Biodeteriodation \& Biodegradation, 40, 269-273.

Smith, Ll., 1941, Weather pits in granite of the Southern Piedmont: Journal of Geomorphology, 4, 117-127.

Smith, B.J., 1977, Rock temperature measurements from the northwest Sahara and their implications for rock weathering: Catena, 4(1-2), 41-63, DOI: 10.1016/0341-8162(77)90011-X.

Smith, B.J., Srinivasan, S., Gómez-Heras, M., Basheer, P.A.M., Viles, H.A., 2011, Nearsurface temperature cycling of stone and its implications for scales of surface deterioration: Geomorphology, 130(1), 76-82.

Schnepfleitner, H., Sass, O., Fruhmann, S., Viles, H., Goudie, A., 2015, A multi-method investigation of temperature, moisture and salt dynamics in tafoni (Tafraoute, Morocco): Earth Surface Processes and Landforms, 41(4), 473-485, DOI: 10.1002/esp.3838.

Twidale, G.R., 1982, Granite Landforms: Elsevier, Amsterdam, $312 \mathrm{p}$
Twidale, C.R., Bourne, J.A., 1975, The subsurface initiation of some minor granite landforms: Journal of the Geological Society of Australia, 22, 477-484.

Twidale, C.R., Bourne, J.A., 1976, Origin and significance of pitting on granite rocks: Zeitschrift für Geomorphologie, 20, 405-416.

Twidale, G.R., Corbin, E.M., 1963, Gnammas: Revue de Géomorphologie Dynamique, 14, $1-20$.

Uña, E., 1999, Evidencias y pruebas significativas sobre la degradación subaérea en formas menores graníticas: Análisis de Caos. Cuadernos Laboratorio Xeolóxico de Laxe, 24, 177-190.

Vidal Romaní, J.R., 1983, El Cuaternario de la provincia de la Coruña. Geomorfología granítica. Modelos elásticos para formación de cavidades: Madrid, Universidad Complutense de Madrid, tesis doctoral, 600 p.

Vidal Romaní, J.R., 1985, Estudio teórico sobre el origen de las características morfológicas de las pías (gnammas, vasque): Cuadernos Laboratorio Xeolóxico de Laxe, 10, 133-168.

Viles, H.A., Goudie, A.S., 2004, Biofilms and case hardening on sandstones from Al-Quwayra, Jordan: Earth Surface Processes and Landforms, 29, 1473-1485, DOI: 10.1002/ esp. 1134 .

Watson, A., Pye, K., 1985, Pseudokarstic microrelief and other weathering features on the Mswati Granite (Swaziland): Zeitschrift fur Geomorphologie, 29, 285-300.

Wilhelmy, H., 1964, Cavernous rock surfaces in semi-arid and arid climates, Pakistan: Geographical Review, 19(2), 8-13. 